\title{
Косолап А.И.
}

\section{ПРОВЕРКА ЧИСЛЕННОЙ ЭФФЕКТИВНОСТИ МЕТОДОВ ГЛОБАЛЬНОЙ ОПТИМИЗАЦИИ}

\section{ГВУЗ «Украинский государственный химико-технологический университет», г. Днепр, Украина}

\begin{abstract}
В данной работе предложены новые сложные тестовые задачи для проверки численной эффективности методов глобальной оптимизации. Это задачи безусловной оптимизации с неизвестными решениями. Предлагаемые тестовые задачи являются несепарабельными и имеют произвольную размерность. Автор предлагает также включить в список тестовых функций для численной проверки эффективности методов тестовые функции J. Nie. Эти функции также несепарабельные произвольной размерности с неизвестными решениями. Предлагаемые тестовые задачи имеют множество локальных экстремумов. Проверка эффективности методов глобальной оптимизации для таких функций упрощается. Если метод оптимизации позволяет улучшать найденные решения тестовых задач, то он будет более эффективным. Существующие методы глобальной оптимизации сравниваются с методом точной квадратичной регуляризации, который разработан автором. Фактически данный метод сравнивается с известными пакетами программ, реализующих современные методы глобальной оптимизации. Эти пакеты включают несколько методов. Лучшие из них используют выпуклую релаксацию задачи для получения оценок решений с последующим использованием программ локальной оптимизации. Но даже такие мощные пакеты испытывают трудности при решении рассмотренных тестовых задач. Некоторые тестовые задачи, например, с функцией Rana или Egg Holder, решаются разными методами уже более 20 лет. За это время ни один метод не позволил получить результаты, которые получены методом точной квадратичной регуляризации. Практически для всех сложных тестовых задач с неизвестными решениями этот метод позволил получить лучшие решения. Иногда преимущество данного метода было существенным, как это в случае с тестовой функцией Rana. Суть метода точной квадратичной регуляризации заключается в преобразовании любой задачи глобальной оптимизации к максимизации квадрата евклидовой нормы вектора на выпуклом множестве. Такая задача в вычислительном плане значительно проще. Часто при таком преобразовании мультимодальная задача становится унимодальной, которая легко решается.
\end{abstract}

Ключевые слова: тестовые задачи, глобальная оптимизация, унимодальные задачи, мультимодальные задачи, численные методы.

DOI: $10.32434 / 2521-6406-2020-8-2-15-23$

\section{Постановка проблемы}

Задачи оптимизации возникают практически в любой сфере человеческой деятельности: в экономике и финансах, на производстве и в технологических процессах, управлении, проектировании, информатике, искусственном интеллекте и многих других областях. Оптимизация включает две составляющие: построение оптимизационных моделей систем и их численное решение. Большинство моделей включают целевую функцию, зависящую от многих переменных, а также ограничения на эти переменные, которые задаются в виде равенств или неравенств. Такие ограничения могут включать принадлежность переменных заданным множествам, например, множеству целых чисел, булевых или дискретных переменных. Необходимо найти значения этих переменных, при которых целе-

(с) Косолап А.И., 2020 
вая функция будет принимать экстремальное (минимальное или максимальное) значение, а ограничения задачи будут выполняться. Целевая функция и ограничения, как правило, задаются с помощью дифференцируемых функций. Например, очень многие задачи могут быть описаны оптимизационными моделями с квадратичными функциями, которые бесконечно раз дифференцируемые. Это упрощает построение эффективных алгоритмов.

Оптимизационные модели разделяют на классы по их сложности. Это линейные, выпуклые, дискретные и невыпуклые модели. Для их решения разработано десятки различных методов и компьютерных программ. При решении конкретной оптимизационной задачи возникает проблема выбора соответствующего метода для ее решения. Методы численного решения оптимизационных задач можно разделить на два класса. К первому классу относятся методы, которые позволяют находить локальные экстремумы задачи. Таких методов большинство. В конце прошлого века начали разрабатываться методы для поиска глобального экстремума. Наличие большого числа оптимизационных методов ставит проблему их численной эффективности. В 70-х годах прошлого века была разработана теория сходимости методов оптимизации, в частности, скорости их сходимости. Однако практическое воплощение этих исследований было затруднено. Это было связано с тем, что сходимость метода удавалось доказать только при определенных условиях, причем практическое выполнение этих условий составляло проблему. Для обеспечения заданной скорости алгоритма также требовались дополнительные условия, причем на разных итерациях алгоритма скорость сходимости могла быть разной. Значительный ущерб этой теории сходимости был нанесен исследованиями по линейному программированию. В 70-х годах прошлого века был разработан полиномиальный алгоритм эллипсоидов для решения задач линейного программирования. Этот алгоритм теоретически был намного эффективнее существующего симплекс-метода. Однако практические вычисления говорили об обратном. Через некоторое время об алгоритме эллипсоидов практически забыли, а симплексметод продолжали с успехом использовать при решении практических задач. Это вынудило разработчиков новых методов оптимизации искать другие способы проверки эффективности методов. Выход был найден в определении множества тестовых задач и проверке эффективности методов посредством решения этих задач. В настоящее время для решения задач поиска локального экстремума разработаны достаточно эффективные алгоритмы, позволяюшие решать задачи большой размерности, и проверка их эффективности не столь актуальна, как для методов поиска глобального экстремума.

Для задач глобальной оптимизации в настоящее время предлагается огромное число (больше тысячи) тестовых задач. Для разработчиков новых методов возникает проблема систематизации этих тестовых задач. Частично эта проблема решается в рамках данной статьи.

\section{Цель статьи}

Целью настоящей работы является систематизация в области тестовых задач глобальной оптимизации и численная проверка эффективности методов глобальной оптимизации в конечномерном евклидовом пространстве.

Анализ последних исследований и публикаций

В настоящее время для численной проверки эффективности методов глобальной оптимизации разработано большое число тестовых задач. Эти задачи включают трудные тестовые задачи, содержащие большое число локальных экстремумов. Часто это число равно $2^{\mathrm{n}}$ или $\mathrm{n}$ ! локальных экстремумов. Часть тестовых задач предлагается из области приложений. Это задачи оптимального проектирования, химического равновесия атомов, задачи упаковки шарами, задачи теории расписаний и многие другие. Тестовые задачи безусловной оптимизации включены в список Benchmark functions. В работе [1] приведено 175 таких функций. Часть этих функций имеет размерность n, а значительная часть меньшей размерности, в том числе размерности 2. Все задачи в [1] даны с решениями. Для функций размерности $n$ решения приведены для случая $\mathrm{n}=2$. Многие из этих функций являются сепарабельными (являются суммой функций одной переменной), поэтому их решения известны для любого n. Только для несепарабельных функций Rana и Egg Holder из этого списка решения для $\mathrm{n}>2$ неизвестны.

Намного больше известно тестовых задач условной оптимизации. Эти тестовые задачи включены в базы MINLPLib Model Statistics и GLOBALLLib Model Statistics, которые легко найти в Internet $[2,3]$. Эти тестовые задачи, как правило, квадратичные, иногда содержат иррациональные функции. В первой базе почти для всех задач даны решения прямой и двойственной задачи. Во второй базе для части задач даны решения. Размерности этих задач колеблются от 
2 до несколько тысяч переменных, тоже можно сказать и об ограничениях. Эти тестовые задачи решались известными программными пакетами глобальной оптимизации BARON, ANTIGONE, COUENNE, LINDO, SCIP и другими. Эти коммерческие пакеты требуют для решения соответствующего представления данных при помощи языков моделирования GAMS, AMPL и других. BARON (Навигатор оптимизации ветвей и сокращения) - это глобальный решатель MINLP разработан исследовательской группой Н.В. Сахинидиса (Ryoo and Sahinidis 1996; Tawarmalani and Sahinidis 2005) используется преимущественно для решения общих квадратичных задач. LINDO - это программа поиска глобального экстремума, разработанная LINDO Systems Inc. и использующая локальный поиск в сочетании с выпуклой релаксацией задачи, позволяющей попасть в окрестность точки глобального экстремума, где потом используется локальный поиск для уточнения решения. Программа COUENNE с открытым кодом использует выпуклые верхние и нижние огибающие для нелинейного оценивания и предназначена для задач глобальной оптимизации. ANTIGONE (Алгоритмы непрерывной целочисленной глобальной оптимизации нелинейных уравнений) - это решатель глобальной оптимизации, разработанный Р. Мизенером и С.А. Флудас из Принстонского университета. Как глобальный решатель, ANTIGONE не ограничивается только выпуклыми задачами, но также может решать множество невыпуклых задач. Он также использует выпуклые релаксации. Программный пакет SCIP использует многогранные внешние аппроксимации и технику пространственных ветвей и границ. Детальная обзорная информация об этих пакетах и других пакетах глобальной оптимизации представлена в работе [4]. В работе будут использоваться результаты численных экспериментов, полученных этими программами для проверки сравнительной численной эффективности методов глобальной оптимизации. В статье приведены результаты численных экспериментов по решению тестовых задач, полученных методом точной квадратичной регуляризации, который предложенный автором настоящей работы.

\section{Изложение основного материала}

Для объективной оценки численной эффективности методов глобальной оптимизации необходимо провести классификацию тестовых задач. Задачи с известными решениями для численных решений являются не информативны- ми, также как и задачи малой размерности. Более информативными являются задачи с неизвестными решениями. Если для таких задач метод позволяет найти лучшее решение, то это будет свидетельствовать о его большей эффективности. Если рассматривать тестовые задачи безусловной оптимизации, то задач с неизвестной точкой глобального минимума будет только две. Это функция Rana

$$
\begin{aligned}
& \min \left\{\sum _ { \mathrm { i } = 1 } ^ { \mathrm { n } - 1 } \left[\left(\mathrm{x}_{\mathrm{i}+1}+1\right) \cos \left(\sqrt{\left|\mathrm{x}_{\mathrm{i}+1}-\mathrm{x}_{\mathrm{i}}+1\right|}\right) \times\right.\right. \\
& \times \sin \left(\sqrt{\left|\mathrm{x}_{\mathrm{i}+1}+\mathrm{x}_{\mathrm{i}}+1\right|}\right)+\mathrm{x}_{\mathrm{i}} \cos \left(\sqrt{\left|\mathrm{x}_{\mathrm{i}+1}+\mathrm{x}_{\mathrm{i}}+1\right|}\right) \times \\
& \left.\left.\times \sin \left(\sqrt{\left|\mathrm{x}_{\mathrm{i}+1}-\mathrm{x}_{\mathrm{i}}+1\right|}\right)\right] \mid-520 \leq \mathrm{x} \leq 520\right\}
\end{aligned}
$$

и функция Egg Holder

$$
\begin{aligned}
& \min \left\{\sum _ { i = 1 } ^ { n - 1 } \left[-x_{i} \sin \left(\sqrt{\left|x_{i}-x_{i+1}-47\right|}\right)-\right.\right. \\
& \left.-\left(x_{i+1}+47\right) \sin \left(\sqrt{\left|x_{i+1}+x_{i} / 2+47\right|}\right)\right], \\
& -512 \leq x \leq 512\} .
\end{aligned}
$$

Для проверки численной эффективности методов глобальной оптимизации необходимо расширить список тестовых функций с неизвестными решениями. Несколько таких функций были предложены J. Nie [5], но они еще не включены в списки для тестирования. Считаем, что их следует включить для обязательного тестирования методов глобальной оптимизации. Ниже приведены функции (F1, F2, ..., F9) J. Nie, которые могут использоваться для тестирования методов глобальной оптимизации. Эти функции произвольной размерности и поэтому представляют целое семейство тестовых задач (задачи могут решаться для любого n):

$$
\begin{aligned}
& \min \left\{\sum_{1 \leq \mathrm{i}<\mathrm{j}<\mathrm{k}<\mathrm{l} \leq \mathrm{n}}(-\mathrm{i}-\mathrm{j}+\mathrm{k}+1) \mathrm{x}_{\mathrm{i}} \mathrm{x}_{\mathrm{j}} \mathrm{x}_{\mathrm{k}} \mathrm{x}_{1}\|\| \mathrm{x} \|^{2}=1\right\} ; \\
& \min \left\{\sum _ { 1 \leq \mathrm { i } < \mathrm { j } < \mathrm { k } \leq \mathrm { n } } \left(\mathrm{x}_{\mathrm{i}}^{2} \mathrm{x}_{\mathrm{j}}^{2} \mathrm{x}_{\mathrm{k}}^{2}+\mathrm{x}_{\mathrm{i}}^{3} \mathrm{x}_{\mathrm{j}}^{2} \mathrm{x}_{\mathrm{k}}+\mathrm{x}_{\mathrm{i}}^{2} \mathrm{x}_{\mathrm{j}}^{3} \mathrm{x}_{\mathrm{k}}+\right.\right. \\
& \left.\left.+\mathrm{x}_{\mathrm{i}} \mathrm{x}_{\mathrm{j}}^{3} \mathrm{x}_{\mathrm{k}}^{2}\right)\|\| \mathrm{x} \|^{2}=1\right\} ;
\end{aligned}
$$




$$
\begin{aligned}
& \min \left\{\sum _ { 1 \leq i < j < k \leq n } \left(x_{i}^{2} x_{j}^{2} x_{k}^{2}-x_{i}^{3} x_{j}^{2} x_{k}-x_{i}^{2} x_{j}^{3} x_{k}+\right.\right. \\
& \left.\left.+x_{i} x_{j}^{3} x_{k}^{2}\right)\|\mid x\|^{2}=1\right\} ; \\
& \min \left\{\sum_{1 \leq i<j \leq n}\left(x_{i} x_{j}+x_{i}^{2} x_{j}-x_{j}^{3}-x_{i}^{2} x_{j}^{2}\right) \mid-1 \leq x \leq 1\right\} ;
\end{aligned}
$$

$$
\min \left\{\sum_{\mathrm{i}=1}^{\mathrm{n}} \mathrm{x}_{\mathrm{i}}^{4}+\sum_{\mathrm{i}=1}^{\mathrm{n}-2} \mathrm{x}_{\mathrm{i}} \mathrm{x}_{\mathrm{i}+1} \mathrm{x}_{\mathrm{i}+2}\right\}
$$$$
\min \left\{\sum_{i=1}^{n} x_{i}^{6}+2 \sum_{i=1}^{n-1} x_{i}^{3} x_{i+1}^{3}\|x\|^{2}=1\right\}
$$$$
\min \left\{\sum_{\mathrm{k}=1}^{3}\left(\sum_{\mathrm{i}=1}^{\mathrm{n}} \mathrm{x}_{\mathrm{i}}^{\mathrm{k}}-1\right)^{2}+\right.
$$$$
\left.+\sum_{\mathrm{i}=1}^{\mathrm{n}}\left(\mathrm{x}_{\mathrm{i}-1}^{2}+\mathrm{x}_{\mathrm{i}}^{2}+\mathrm{x}_{\mathrm{i}+1}^{2}-\mathrm{x}_{\mathrm{i}}^{3}-1\right)^{2}\right\} \text {; }
$$

$$
\min \left\{\sum_{\mathrm{i}=1}^{\mathrm{n}} \mathrm{x}_{\mathrm{i}}+\sum_{1 \leq \mathrm{i}<\mathrm{j} \leq \mathrm{n}}^{\mathrm{n}} \mathrm{x}_{\mathrm{i}} \mathrm{x}_{\mathrm{j}}+\sum_{1 \leq \mathrm{i}<\mathrm{j}<\mathrm{k} \leq \mathrm{n}}^{\mathrm{n}} \mathrm{x}_{\mathrm{i}} \mathrm{x}_{\mathrm{j}} \mathrm{x}_{\mathrm{k}}+\right.
$$$$
\left.+\sum_{1 \leq i<j<k<1 \leq n}^{n} x_{i} x_{j} x_{k} x_{1}\|x\|^{2} \leq 1\right\} \text {; }
$$

$$
\min \left\{\sum_{1 \leq i<j<k \leq n}^{n}\left((i+j) x_{i} x_{j} x_{k}+(j+k) x_{i}^{2} x_{j}^{2} x_{k}^{2}\right) \mid \sum_{i=1}^{n} x_{i}^{4} \leq 1\right\} .
$$

К этим функциям следует добавить задачи P1, P2 J. Nie с простыми ограничениями:

$$
\begin{aligned}
& \min \left\{\sum _ { 1 \leq \mathrm { i } < \mathrm { j } < \mathrm { k } \leq \mathrm { n } / 2 } \left(\mathrm{x}_{\mathrm{i}} \mathrm{x}_{\mathrm{j}} \mathrm{x}_{\mathrm{k}}+\mathrm{x}_{\frac{\mathrm{n}}{2}+\mathrm{i}} \mathrm{x}_{\frac{\mathrm{n}}{2}+\mathrm{j}} \mathrm{x}_{\frac{\mathrm{n}}{2}+\mathrm{k}}+\right.\right. \\
& \left.\left.+\mathrm{x}_{\mathrm{i}} \mathrm{x}_{\mathrm{j}} \mathrm{x}_{\mathrm{k}} \mathrm{x}_{\frac{\mathrm{n}}{2}+\mathrm{i}} \mathrm{x}_{\frac{\mathrm{n}}{2}+\mathrm{j}} \mathrm{x}_{\frac{\mathrm{n}}{2}+\mathrm{k}}\right)\right\}, \\
& \mathrm{x}_{1}^{4}+\ldots+\mathrm{x}_{\frac{\mathrm{n}}{2}}^{4} \leq 1, \quad \mathrm{x}_{\frac{\mathrm{n}}{2}}^{4}+\ldots+\mathrm{x}_{\mathrm{n}}^{4} \leq 1 ;
\end{aligned}
$$

$$
\begin{aligned}
& \min \left\{\sum _ { 1 \leq i < j < k \leq n / 2 } \left(x_{i} x_{j} x_{k}\left(1+x_{i}+x_{j}+x_{k}\right)+\right.\right. \\
& \left.\left.+i x_{i}^{6}+j x_{j}^{6}+k x_{k}^{6}\right)\right\}, \\
& \quad x_{1}^{4}+\ldots+x_{\frac{n}{2}}^{4} \leq 1, \quad x_{\frac{n}{2}+1}^{4}+\ldots+x_{n}^{4} \leq 1 .
\end{aligned}
$$

Видим, что все эти функции являются несепарабельными и точное значение глобального минимума в них неизвестно. Также была модифицирована функция Zakharova (M. Zakharov)

$$
\begin{aligned}
& \min \left\{\sum_{\mathrm{i}=1}^{\mathrm{n}} \mathrm{x}_{\mathrm{i}}+\left(0,5 \sum_{\mathrm{i}=1}^{\mathrm{n}} \mathrm{ix} \mathrm{x}_{\mathrm{i}}\right)^{2}+\right. \\
& \left.+\left(0,5 \sum_{\mathrm{i}=1}^{\mathrm{n}} \mathrm{ix}_{\mathrm{i}}\right)^{4} \mid \mathrm{x} \in[-5,10]\right\},
\end{aligned}
$$

решение которой тривиально $\mathrm{x}=0$ на функцию

$$
\begin{aligned}
& \min \left\{-\sum_{i=1}^{n} x_{i}-\left(0,5 \sum_{i=1}^{n} i x_{i}\right)^{2}+\right. \\
& \left.+\left(0,5 \sum_{i=1}^{n} i x_{i}\right)^{4} \mid x \in[-5,10]\right\},
\end{aligned}
$$

точное решение которой теперь неизвестно. Далее, в списке Benchmark functions были обобщены некоторые несепарабельные функции двух переменных на функции $\mathrm{n}$ переменных. После такого обобщения их решение неизвестно для $\mathrm{n}>2$. Это такие функции, как функция Adjman

$\min \left\{\sum_{\mathrm{i}=1}^{\mathrm{n}-1} \cos \left(\mathrm{x}_{\mathrm{i}}\right) \sin \left(\mathrm{x}_{\mathrm{i}+1}\right)+\sum_{\mathrm{i}=1}^{\mathrm{n}} \frac{\mathrm{x}_{\mathrm{i}}}{\mathrm{x}_{\mathrm{i}+1}^{2}+1} \mid \mathrm{x} \in[-1,1]\right\}$;

функция Bird

$$
\begin{aligned}
& \min \left\{\sum _ { \mathrm { i } = 1 } ^ { \mathrm { n } - 1 } \left[\sin \left(\mathrm{x}_{\mathrm{i}}\right) \mathrm{e}^{\left(1-\cos \left(\mathrm{x}_{\mathrm{i}+1}\right)\right)^{2}}+\cos \left(\mathrm{x}_{\mathrm{i}+1}\right) \mathrm{e}^{\left(1-\sin \left(\mathrm{x}_{\mathrm{i}}\right)\right)^{2}}+\right.\right. \\
& \left.\left.+\left(\mathrm{x}_{\mathrm{i}}-\mathrm{x}_{\mathrm{i}+1}\right)^{2}\right] \mid \mathrm{x} \in[-2 \pi, 2 \pi]\right\} ;
\end{aligned}
$$


функция Mishra 6

$$
\begin{aligned}
& \min \left\{\sum_{\mathrm{i}=1}^{\mathrm{n}-1}-\ln \left[\sin ^{2}\left(\cos \left(\mathrm{x}_{\mathrm{i}}\right)+\cos \left(\mathrm{x}_{\mathrm{i}+1}\right)\right)^{2}-\right.\right. \\
& \left.-\cos ^{2}\left(\sin \left(\mathrm{x}_{\mathrm{i}}\right)+\sin \left(\mathrm{x}_{\mathrm{i}+1}\right)\right)+\mathrm{x}_{\mathrm{i}}\right]^{2}+ \\
& \left.+0,001\left(\left(\mathrm{x}_{\mathrm{i}}-1\right)^{2}+\left(\mathrm{x}_{\mathrm{i}+1}-1\right)^{2}\right)\right\} ;
\end{aligned}
$$

функция Scahffer 4

$$
\begin{aligned}
& \min \left\{\sum_{i=1}^{n-1}\left(0,5+\frac{\cos ^{2}\left(\sin \left(x_{i}^{2}-x_{i+1}^{2}\right)-0,5\right)}{1+0,001\left(x_{i}^{2}+x_{i+1}^{2}\right)^{2}}\right) \mid\right. \\
& x \in[-100,100]\} ;
\end{aligned}
$$

функция Trefethen

$$
\begin{aligned}
& \min \left\{\sum _ { \mathrm { i } = 1 } ^ { \mathrm { n } - 1 } \left[\mathrm{e}^{\sin \left(50 \mathrm{x}_{\mathrm{i}}\right)}+\sin \left(60 \mathrm{x}_{\mathrm{i}+1}\right)+\sin \left(70 \sin \left(\mathrm{x}_{\mathrm{i}}\right)\right)+\right.\right. \\
& +\sin \left(\sin \left(80 \mathrm{x}_{\mathrm{i}+1}\right)\right)-\sin \left(10\left(\mathrm{x}_{\mathrm{i}}+\mathrm{x}_{\mathrm{i}+1}\right)\right)+ \\
& \left.\left.+0,25\left(\mathrm{x}_{\mathrm{i}}^{2}+\mathrm{x}_{\mathrm{i}+1}^{2}\right)^{2}\right] \mid \mathrm{x} \in[-10,10]\right\} .
\end{aligned}
$$

Все эти функции являются несепарабельными и точное их решение для $\mathrm{n}>2$ неизвестно. $\mathrm{K}$ тестовой задаче безусловной оптимизации относят также поиск минимальной потенциальной системы атомов, в которых находят координаты центров атомов или молекул, при которых потенциальная энергия атомов будет минимальной. Уже для 5 атомов точное значение потенциальной энергии:

$$
\begin{gathered}
\min \left\{4 \sum_{\mathrm{i}<\mathrm{j}}\left(\frac{1}{\mathrm{r}_{\mathrm{ij}}^{12}}-\frac{1}{\mathrm{r}_{\mathrm{ij}}^{6}}\right)\right\}, \\
\mathrm{r}_{\mathrm{ij}}=\sqrt{\left(\mathrm{x}_{\mathrm{i}}-\mathrm{x}_{\mathrm{j}}\right)^{2}+\left(\mathrm{y}_{\mathrm{i}}-\mathrm{y}_{\mathrm{j}}\right)^{2}+\left(\mathrm{z}_{\mathrm{i}}-\mathrm{z}_{\mathrm{j}}\right)^{2}}
\end{gathered}
$$

неизвестно. В базе данных doye.chem.ox.as.uk содержатся данные о решении задачи с числом атомов до 1000. Эта задача является сложной для численного решения и содержит множество локальных минимумов. Есть еще некоторые ме- нее известные тестовые функции, например:

$$
\min \left\{\sum_{\mathrm{i}=1}^{\mathrm{n}-1}\left(\sin \left(\mathrm{x}_{\mathrm{i}}+\mathrm{x}_{\mathrm{i}+1}\right)+\sin \left(\frac{2 \mathrm{x}_{\mathrm{i}} \mathrm{x}_{\mathrm{i}+1}}{3}\right)\right) \mid 3 \leq \mathrm{x} \leq 13\right\}
$$

с неизвестным решением. Для более полного исследования численной эффективности методов оптимизации этот список тестовых функций необходимо увеличить. Однако и этот список достаточно большой, так как каждая задача может решаться для любого $\mathrm{n}$.

Среди тестовых задач условной оптимизации преобладают задачи с неизвестными решениями. Однако численные эксперименты для этого класса задач менее информативны. Для этих задач необходимо учитывать точность выполнения ограничений. Иногда незначительное изменение точности выполнения одного или нескольких ограничений может привести к существенному изменению значения целевой функции. За исключением приведенных выше баз данных в публикациях, как правило, не приводятся найденные решения (точки глобального минимума), что не дает возможность проверить точность ограничений. Можно допустить, что два решения тестовой задачи условной минимизации различаются, если они приводят к различным локальным минимумам.

Тестовые задачи условной оптимизации имеют, как правило, фиксированную размерность. Но некоторые задачи с простыми ограничениями могут иметь и произвольную размерность. Например, такой является достаточно известная тестовая задача Keane:

$$
\begin{aligned}
& \max \left\{\mid \frac{\sum_{\mathrm{i}=1}^{\mathrm{n}} \cos ^{4}\left(\mathrm{x}_{\mathrm{i}}\right)-2 \prod_{\mathrm{i}=1}^{\mathrm{n}} \cos ^{2}\left(\mathrm{x}_{\mathrm{i}}\right)}{\sqrt{\sum_{\mathrm{i}=1}^{\mathrm{n}} \mathrm{i} \mathrm{x}_{\mathrm{i}}^{2}}}\right\}, \\
& 0,75-\prod_{\mathrm{i}=1}^{\mathrm{n}} \mathrm{x}_{\mathrm{i}} \leq 0, \sum_{\mathrm{i}=-1}^{\mathrm{n}} \mathrm{x}_{\mathrm{i}}-7,5 \mathrm{n} \leq 0, \\
& 0 \leq \mathrm{x}_{\mathrm{i}} \leq 10, \mathrm{i}=1, \ldots, \mathrm{n} .
\end{aligned}
$$

Существует достаточно сложный класс задач упаковки некоторых областей (квадрата, круга, правильного треугольника) кругами одного или разных диаметров. Такие задачи возникают при проектировании электронных схем. В этих задачах ищется наиболее плотная упа- 
ковка областей кругами. В литературе приведены данные о решенных задачах различной размерности [6] без указания центров кругов. Еще одной сложной задачей является квадратичная задача о назначениях, которая находит самые разные применения. Для этой задачи разработаны тестовые примеры и приведены найденные решения [7]. Среди задач разной размерности можно указать задачу построения многоугольника максимальной площади с заданным количеством сторон и радиусом единица. Это тоже очень сложная задача для численного решения и ее размерность зависит от числа сторон многоугольника. Автором была решена такая задача для многогранника с 20 сторонами. Это задача с большим числом ограничений. Точность выполнения ограничений в точке минимума порядка $10^{-8}$. Результаты решения показаны в таблице под номером 18. Достаточно важными и одновременно сложными для численного решения являются задачи теории расписания, они относятся к общим квадратичным задачам. Автору неизвестны тестовые примеры для решения этого класса задач.

При проведении численных экспериментов для проверки эффективности методов важна размерность задачи. Для задач глобальной оптимизации достаточной размерностью является $n=100$. Обычно в публикациях приводится значительно меньшая размерность тестовых задач. Так как в последних публикациях по глобальной оптимизации приводятся лучшие результаты по решению тестовых задач, то автор использовал эти результаты для сравнения. Для новых тестовых задач, предложенных автором, использовался метод эволюционного поиска из библиотеки Python.

Остановимся на методах решения задач глобальной оптимизации. В общем случае эти задачи можно записать в виде:

$$
\min \left\{f_{0}(x) \mid f_{i}(x) \leq 0, i=1, \ldots, m, x \in E^{n}\right\},
$$

где x - искомый n-мерный вектор (точка) евклидового пространства $\mathrm{E}^{\mathrm{n}}$.

Первыми методами для решения задач глобальной оптимизации вида (1) были методы мультистарта. В этих методах выбирается большое число начальных точек, равномерно покрывающее допустимую область задачи, и для каждой такой точки решается задача (1) методом локальной оптимизации. Среди найденных решений выбирается решение с наименьшим значением целевой функции. Однако такой метод в n-мерном пространстве является неэффективным уже при $\mathrm{n}>10$. Если предположить, что выбирается только одна точка в каждом ортанте системы координат, то потребуется выбирать $2^{\mathrm{n}}$ точек. Для многих начальных точек этим методом получают одно и то же решение, а возможность покрыть достаточно плотно допустимое множество уже для 10-мерного пространства невозможно. Кроме того, допустимые области мультимодальных задач имеют довольно сложную структуру и иногда даже найти одну допустимую точку является вычислительной проблемой. Практические задачи требуют решения в пространстве размерности сотен и десятки тысяч переменных. В дальнейшем метод мультистарта был модифицирован в генетические и эволюционные алгоритмы и последовательность начальных точек выбиралась случайным образом в соответствии с некоторым алгоритмом. Эти алгоритмы не намного эффективнее мультистарта. Современные программные пакеты глобальной оптимизации включают несколько различных методов. Большинство из них используют выпуклую релаксацию для упрощения задачи и получения приближенных решений, верхних или нижних оценок решений. Затем полученные решения используют для локального поиска.

Иной подход используется в методе точной квадратичной регуляризации [8]. Точная квадратичная регуляризация позволяет преобразовать исходную задачу (1) к более простой задаче поиска максимума квадрата нормы вектора на выпуклом множестве:

$$
\begin{aligned}
& \max \left\{\|x\|^{2} \mid f_{0}(x)+s+(r-1)\|x\|^{2} \leq d,\right. \\
& \left.f_{i}(x)+r\|x\|^{2} \leq d, i=1, \ldots, m, \quad x \in E^{n+1}\right\},
\end{aligned}
$$

где $\|x\|^{2}-$ квадрат евклидовой нормы вектора, $\|\mathrm{x}\|^{2}=\mathrm{x}_{1}{ }^{2}+\ldots+\mathrm{x}_{\mathrm{n}}{ }^{2}+\mathrm{x}_{\mathrm{n}+1}{ }^{2}$. Таким образом, при преобразовании размерность вектора х увеличивается на единицу. Преобразованная задача (2) использует также два параметра $\mathrm{r}$ и $\mathrm{s}$, где параметр r>0 выбирается таким, чтобы допустимое множество задачи (2) было выпуклым, а параметр s таким, чтобы первое ограничение задачи (2) в точке максимума было активным. Кроме того, преобразованная задача (2) содержит еще одну новую переменную d, значение которой находим методом дихотомии. Последовательно увеличивая значение переменной $\mathrm{d}$, для каждого фиксированного ее значения решаем задачу (2) прямо-двойственным методом внут-

Kosolap A.I. 
ренней точки. Найденную точку х проверяем на выполнимость условия $\mathrm{r}\|\mathrm{x}\|^{2}=\mathrm{d}$. Если это условие выполняется с заданной точностью и значение d минимально, то решение задачи (2) совпадает с решением задачи (1).

Для программной реализации метода точной квадратичной регуляризации необходима только программа поиска локального экстремума, реализующая прямо-двойственный метод внутренней точки [9].

Рассмотрим алгоритм данного метода, который использовался в численных экспериментах.

Алгоритм.

Шаг 1. Выбираем значения параметров $\mathrm{r}$ и $\mathrm{s}$ удовлетворяющие приведенным выше условиям.

Шаг 2. Находим минимальное значение переменной d, решая выпуклую задачу

$$
\begin{aligned}
& \min \left\{d \mid f_{0}(x)+s+(r-1)\|x\|^{2} \leq d\right. \\
& \left.f_{i}(x)+r\|x\|^{2} \leq d, \quad i=1, \ldots, m\right\} .
\end{aligned}
$$

Эта задача всегда будет иметь решение. Решением данной задачи будет точка $\left(\mathrm{x}^{0}, \mathrm{~d}_{0}\right)$.

Если в этой точке выполняется условие $\mathrm{r}\left\|\mathrm{x}^{0}\right\|^{2}=\mathrm{d}_{0}$, то задача (1) решена и $\mathrm{x}^{0}-$ ее решение. В противном случае переходим к следующему шагу.

Шаг 3. Если $\mathrm{r}\left\|\mathrm{x}^{0}\right\|^{2}<\mathrm{d}_{0}$, то выбираем шаг изменения переменной $\mathrm{d}_{\mathrm{k}+1}=\mathrm{d}_{\mathrm{k}}+\mathrm{Dd}_{\mathrm{k}}$, где величина шага $\mathrm{Dd}_{\mathrm{k}}$ зависит от величины $\mathrm{r}\left\|\mathrm{x}^{\mathrm{k}}\right\|^{2}-\mathrm{d}_{\mathrm{k}}$. Чем больше эта разность по абсолютной величине, тем больше шаг изменения $\mathrm{d}_{\mathrm{k}}$. Для каждого фиксированного значения $\mathrm{d}_{\mathrm{k}}$ решаем задачу (2), получаем последовательность решений $\left(\mathrm{x}^{\mathrm{k}}, \mathrm{d}_{\mathrm{k}}\right)$ до достижения равенства $\mathrm{r}\left\|\mathrm{x}^{\mathrm{k}}\right\|^{2}-\mathrm{d}_{\mathrm{k}}$ с заданной точностью. Заметим, что если $\mathrm{r}\left\|\mathrm{x}^{\mathrm{k}}\right\|^{2}-\mathrm{d}$ положительно, то значение $\mathrm{Dd}_{\mathrm{k}}$ выбираем отрицательным. Важно, что при увеличении значения $\mathrm{d}$ предыдущее решение задачи (2) будет допустимым для нового d.

Шаг 4. Если в найденном решении $\left(\mathrm{x}^{*}, \mathrm{~d}^{*}\right)$ значение $\mathrm{d}^{*}$ будет минимально возможным, то $\mathrm{x}^{*}$ - решение задачи (1). Для проверки минимальности $\mathrm{d}^{*}$ будем уменьшать значение $\mathrm{d}^{*}$ малым шагом. Если при таком изменении $d$ разность $\mathrm{r}\left\|\mathrm{x}^{\mathrm{k}}\right\|^{2}-\mathrm{d}_{\mathrm{k}}$ будет отрицательной и возрастать по модулю, то $\mathrm{x}^{*}$ - решение задачи (1). В противном случае, если при некотором $\mathrm{d}<\mathrm{d}^{*}$ разность $\mathrm{r}\left\|\mathrm{x}^{\mathrm{k}}\right\|^{2}-\mathrm{d}_{\mathrm{k}}$ будет больше нуля, то дальнейшее уменьшение $\mathrm{d}$ приведет в точку $\left(\mathrm{x}^{\mathrm{k}}, \mathrm{d}_{\mathrm{k}}\right)$, для которой выполняется условие $\mathrm{r}\left\|\mathrm{x}^{\mathrm{k}}\right\|^{2}=\mathrm{d}_{\mathrm{k}}$. Этому значению $\mathrm{x}^{\mathrm{k}}$ будет соответствовать меньшее значение $\mathrm{f}_{0}\left(\mathrm{x}^{\mathrm{k}}\right)$. Этот процесс уменьшения $d$ продолжается до тех пор, пока значение разности $\mathrm{r}\left\|\mathrm{x}^{\mathrm{k}}\right\|^{2}-\mathrm{d}_{\mathrm{k}}$ будет монотонно убывать. Этим завершается процесс минимизации $\mathrm{d}=\mathrm{d}^{*}$, для которого $\mathrm{x}^{*}$ будет решением задачи (1).

Эффективность рассмотренного метода точной квадратичной регуляризации подтверждается многочисленными сравнительными эКспериментами при решении сложных тестовых задач. В таблице приведены результаты числен-

\begin{tabular}{|c|c|c|c|c|}
\hline Задача & $\mathrm{n}$ & $\mathrm{m}$ & Метод EQR & Лучшее известное решение \\
\hline Egg Holder & 100 & 0 & $-89948,532$ & -89938 \\
\hline Rana & 100 & 0 & $-50865,131$ & $-41047,18$ \\
\hline Trefethen & 100 & 0 & $-237,73323$ & - \\
\hline Mishra 6 & 100 & 0 & $-289,8371385$ & $-279,5928$ (python) \\
\hline Adjman & 100 & 0 & $-23,95135$ & $-23,30464$ (python) \\
\hline Scahffer 4 & 100 & 0 & 28,95141 & 48,951266 (python) \\
\hline M. Zakarov & 100 & 0 & $-255133,9934$ & $-254808,9343$ (python) \\
\hline Scahffer 4 & 100 & 0 & 28,95141 & 48,951266 (python) \\
\hline Nie F1 & 50 & 1 & $-0,436768488$ & $>-140,4051$ \\
\hline Nie F2 & 20 & 1 & $-0,058932927$ & $-0,0202$ \\
\hline Nie F3 & 20 & 1 & $-0,0713718$ & 0,02854862 \\
\hline Nie F4 & 50 & 0 & -94 & $>-1250(-26)$ \\
\hline Nie F5 & 20 & 0 & $-1,61914837$ & 0 \\
\hline Nie F6 & 20 & 1 & $-0,048277304$ & 0 \\
\hline Nie F7 & 16 & 0 & 7,812451 & $>7,5586$ \\
\hline Nie F8 & 50 & 1 & $-0,9775492$ & $-0,88776$ \\
\hline Nie F9 & 15 & 1 & $-25,242116$ & $-23,6740182$ \\
\hline Polygon & 40 & 189 & $-0,92788759$ & 0,776859 \\
\hline
\end{tabular}

Результаты решений тестовых задач 
ных экспериментов для достаточно больших размерностей тестовых задач.

В таблице видим, что полученные результаты методом точной квадратичной регуляризации (EQR) значительно превосходят численные результаты всех других методов (в задачах необходимо было найти точку глобального минимума). Автором были проведены масштабные численные эксперименты с 400 известными сложными тестовыми задачами. Эти результаты приведены в книге автора [10] и они также подтверждают эффективность метода EQR.

\section{Выводы}

В данной работе расширен класс тестовых задач для проверки численной эффективности методов глобальной оптимизации. Предложены несепарабельные мультимодальные тестовые задачи произвольной размерности с неизвестными решениями. Проверка эффективности методов глобальной оптимизации для таких тестовых задач упрощается. Если метод находит лучшие решения для класса тестовых задач, то он будет более эффективным. Представлен алгоритм метода точной квадратичной регуляризации, который использовался в численных экспериментах.

Проведены сравнительные численные эксперименты на тестовых задачах достаточно большой размерности, которые подтверждают численное преимущество метода точной квадратичной регуляризации.

\section{СПИСОК ЛИТЕРАТУРЫ}

1. Jamil M, Yang X.S. A literature survey of benchmark functions for global optimization problems // Int. J. Math. Model Numer. Optim. - Vol. 4. - №. 2. - 2013. - P.150-194. https://doi.org/10.1504/IJMMNO.2013.055204.

2. MINLPLib Model Statistics. Режим доступу: https://www.minlplib.org.

3. GLOBALLLib Model Statistics. Режим доступу: https://www.gamsworld.org.

4. A review and comparison of solvers for convex MINLP / J. Kronqvist, D.E. Bernal, A. Lundell, I.E. Grossmann // Optimization and Engineering. - Vol. 20. - 2019. - P.397-455. https://doi.org/10.1007/s11081-018-9411-8.

5. Nie J., Wang L. Regularization methods for SDP relaxations in large-scale polynomial optimization // SIAM Journal on Optimization. - Vol. 22. - 2012. - P.408-428.

https://doi.org/10.1137/110825844.
6. New Approaches to Circle Packing in a Square / P.G. Szabo, M.Cs. Markot, T. Csendes, E. Specht, L.G. Casado, I. Garcia. - Springer, 2007. - 237 p.

7. A Quadratic Assignment Problem Library. Режим доступу: https://anjos.mgi.polymtl.ca/qaplib.

8. Косолап А.И. Глобальная оптимизация. Метод точной квадратичной регуляризации. - Д.: ПГАСА, 2015. - 164 с.

9. Nocedal J., Wright S.J. Numerical optimization. Springer, 2006. $-685 \mathrm{p}$.

10. Kosolap A. Practical Global Optimization. - D.: Publisher Bila K.O., 2020. - 192 p.

Поступила в редакцию 29.11.2020

\section{ПЕРЕВІРКА ОБЧИСЛЮВАЛЬНОӤ ЕФЕКТИВНОСТІ МЕТОДІВ ГЛОБАЛЬНОЇ ОПТИМІЗАЦІЇ}

\section{Косолап A.I.}

У даній роботі запропоновані нові складні тестові задачі для перевірки обчислювальної ефективності методів глобальної оптимізації. Це задачі безумовної оптимізації з невідомими розв'язками. Запропоновані тестові задачі є несепарабельні та мають довільну розмірність. Автор пропонує включити в список тестових функцій для чисельної перевірки ефективності методів тестові функції J. Niе. Ці функції також несепарабельні довільної розмірності з невідомими розв 'язками. Запропоновані тестові задачі мають безліч локальних екстремумів. Перевірка ефективності методів глобальної оптимізації для таких функцій спрощується. Якщо метод дозволяє поліпшити знайдені розв'язки тестових задач, то він буде більш ефективним. Існуючі методи глобальної оптимізації порівнюються з методом точної квадратичної регуляризації, який розроблений автором. Фактично даний метод порівнюється з відомими пакетами програм, що реалізують сучасні методи глобальної оптимізації. Ці пакети включають кілька методів. Кращі з них використовують опуклу релаксацію задачі для отримання оцінок розв'язків з подальшим використанням програм локальної оптимізації. Але навіть такі потужні пакети зазнають труднощів при розв'язуванні розглянутих тестових задач. Деякі тестові задачі, наприклад, з функцією Rапа або Egg Holder, розв'язуються різними методами вже більше 20 років. За цей час жоден метод не дозволив отримати результати, які отримані методом точної квадратичної регуляризації. Практично для всіх складних тестових задач з невідомими розв'язками цей метод дозволив отримати кращі розв'язки. Іноді перевага даного методу була істотною, як це у випадку з тестової функцією Rana. Суть методу точної квадратичної регуляризації полягає в перетворенні будь-якої задачі глобальної оптимізації до максимізації квадрата евклідової норми вектора на опуклій множині. Такі задачі в обчислювальному плані значно простіші. Часто при такому перетворенні мультимодальна задача стає унімодальною, яка легко розв'язується.

Ключові слова: тестові задачі, глобальна оптимізація, унімодальні задачі, мультимодальні задачі, чисельні методи.

Kosolap A.I. 


\section{NUMERICAL VERIFICATION OF THE EFFICIENCY OF} GLOBAL OPTIMIZATION METHODS

Kosolap A.I.

Ukrainian State University of Chemical Technology, Dnipro, Ukraine

In this paper, new difficult test problems are proposed to test the numerical efficiency of global optimization methods. These are problems of unconstrained optimization with unknown solutions. The proposed test problems are inseparable and have arbitrary dimensions. The author also proposes to include the test functions by J. Nie in the list of test functions for numerical verification of the effectiveness of methods. These functions are also inseparable functions of arbitrary dimensions with unknown solutions. The proposed test problems have many local extrema. Testing the effectiveness of global optimization methods for such functions is simplified. If the method allows improving the found solutions to test problems, then it will be more effective. The existing global optimization methods are compared with the exact quadratic regularization method developed by the author. This method is compared with known software packages that implement modern methods of global optimization. These packages include several methods. The best of them use convex relaxation of the problem to obtain estimates of solutions with subsequent use of local optimization programs. But even such powerful packages have difficulties in solving the considered test problems. Some test problems, for example, with the Rana or Egg Holder function, have been solved by different methods for over 20 years. During this time, no method has allowed obtaining results that are obtained by the method of exact quadratic regularization. For almost all complex test problems with unknown solutions, this method yielded better solutions. Sometimes the advantage of this method was significant, as is the case with the Rana test function. The essence of the exact quadratic regularization method is to transform any global optimization problem to maximize the square of the Euclidean norm of a vector on a convex set. This problem is computationally much simpler. Often, with such a transformation, the multimodal problem becomes unimodal, which is easy to solve.

Keywords: test problems, global optimization, unimodal problems, multimodal problems, numerical methods.

\section{REFERENCES}

1. Jamil M., Yang X.S. A literature survey of benchmark functions for global optimization problems. Int. J. Math. Model Numer. Optim. 2013, vol. 4, no. 2, pp.150-194. https://doi.org/10.1504/IJMMNO.2013.055204.

2. MINLPLib Model Statistics, available at: https://www.minlplib.org.

3. GLOBALLLib Model Statistics, available at: https://www.gamsworld.org.

4. Kronqvist J., Bernal D.E., Lundell A., Grossmann I.E. A review and comparison of solvers for convex MINLP. Optimization and Engineering. 2019, vol. 20, pp.397-455. https://doi.org/10.1007/s11081-018-9411-8.

5. Nie J., Wang L. Regularization methods for SDP relaxations in large-scale polynomial optimization. SIAM Journal on Optimization. 2012, vol. 22, pp.408-428.

https://doi.org/10.1137/110825844.

6. Szabo P.G., Markot M.Cs., Csendes T., Specht E., Casado L.G., Garcia I. New Approaches to Circle Packing in a Square. Springer, 2007. 237 p.

7. A Quadratic Assignment Problem Library, available at: https://anjos.mgi.polymtl.ca/qaplib.

8. Kosolap A.I. Globalnaya optimizatciya. Metod tochnoy kvadratichnoy regulyarizatcii. [Global optimization. Method of exact quadratic regularization]. Dnepropetrovsk: PGACA, 2015, 164 p. (in Russian).

9. Nocedal J., Wright S.J. Numerical optimization. Springer, 2006, $685 \mathrm{p}$

10. Kosolap A. Practical Global Optimization. Dnipro: Publisher Bila K.O., 2020, 192 p. 